\title{
Fiber-Optic Based, Force and Torque Compliant Sensing Calibration Strategies
}

\author{
Osama Al-Mai, Jacques Albert, Mojtaba Ahmadi \\ Carleton University \\ 1125 Colonel By Dr, Ottawa, Canada \\ osamalamai@cmail.carleton.ca; jacquesalbert@cunet.carleton.ca; mojtabaahmadi@cunet.carleton.ca
}

\section{Extended Abstract}

With modern biomechanical applications varying from walking assistive devices [1] to human-machine interface [2], compliant sensors are increasingly developed for use in making human interactions more comfortable and safer. Previous sensing approaches, based on electromechanical systems technologies, have the drawback of only measuring one component of the applied force. On the other hand, the strain gauge-based sensors (such as the commercially available ATI 6-axis F/T sensors) are too rigid, bulky and heavy for human applications and may cause fatigue or discomfort. Therefore, new sensing technologies are required that can offer precision in measurement, while offering compliance at the contact points with user. To overcome these issues, we have proposed various compliant fiber-optic based force and torque sensors that have proved their capabilities to accurately measure force and torque in three and six directions. The first prototype is 3 -axis force sensor, composed of three fiber-optic sensing elements. Each element incorporates a pair of receiving and transmitting fibres, lightto voltage convertor (LVT) and a reflecting surface (mirror) affixed between the sensor's top and bottom plates that are separated by rubber blocks providing flexibility [3]. When a certain amount of force is applied along the top surface of the sensor a deflection is generated and change the light intensity occurs representing that applied force. The sensor has small an RMSE \% of $1.92 \%$ with high measurement range of up to $1 \mathrm{kN}$. We have also developed and present a 6-axis F/T sensor by adding three more optical transducers to capture the thee torques. Several calibration methodologies have been used including least squares optimization method, resulting in very good sensing performance [4]. The RMSE is $1.65 \%$ with a fitting value of up to 99\%; however, the transducer exhibits nonlinear dynamic behaviour particularly in case of high forces, which could potentially generate complex errors such as hysteresis. Therefore, we propose in this work, a novel dynamic calibration method using system identification process which is able to reduce the generated hysteresis by more than half compared to the conventional least squares calibration approach.

The higher sensor flexibility may adversely affect its bandwidth; thus, we have investigated the sensor bandwidth and its response to dynamic loading. A second order transfer function was obtained using a data-driven identification process which indicated that the bandwidth of the sensor, when this transfer function is used as an estimation model, is found to be $65 \mathrm{~Hz}$. This is suitable for assistive devices, human-machine interface, robotic rehibition systems, or wearable gait measurements.

\section{References}

[1] J. Li, B. Shen, C.-M. Chew, C. L. Teo, and A. N. Poo, "Novel Functional Task-Based Gait Assistance Control of Lower Extremity Assistive Device for Level Walking," IEEE Transactions on Industrial Electronics, vol. 63, no. 2, pp. 10961106, 2016.

[2] J. Schneider, W. Stork, S. Irgenfried, and H. Worn, "A multimodal human machine interface for a robotic mobility aid," 2015 6th International Conference on Automation, Robotics and Applications (ICARA), 2015.

[3] O. Al-Mai, M. Ahmadi, and J. Albert, "A Compliant 3-Axis Fiber-Optic Force Sensor for Biomechanical Measurement,” IEEE Sensors Journal, vol. 17, no. 20, pp. 6549-6557, 2017.

[4] O. Al-Mai, M. Ahmadi, and J. Albert, "Design, Development and Calibration of a Lightweight, Compliant Six-Axis Optical Force/Torque Sensor,” IEEE Sensors Journal, vol. 18, no. 17, pp. 7005-7014, 2018. 